\title{
The Role of Bipolar Radiofrequency (Bprf) as a Mechanism of Bio-Stimulation for the Treatment of Vulvo-Vaginal Laxity: A Novel Approach
}

\author{
Pablo González Isaza ${ }^{1}$, Diana Velez Rizo², Ignacio Garibay ${ }^{3}$, Ricardo Galvan ${ }^{4, *}$ \\ ${ }^{1}$ Division of Urogynecology and Pelvic Reconstructive Surgery, Department of Obstetrics and Gynecology, San Jorge University \\ Hospital, Pereira, Colombia. \\ ${ }^{2}$ Department of Women Health, Cardioinfantil Foundation, Bogotá, Colombia. \\ ${ }^{3}$ Institute of Laser and Pulsed Light of Mexico, Ignacio, Mexico. \\ ${ }^{4}$ Md Dermatologist, Laser Specialist, Guadalajara, Mexico.
}

\begin{abstract}
How to cite this paper: Pablo González Isaza, Diana Velez Rizo, Ignacio Garibay, Ricardo Galvan. (2021) The Role of Bipolar Radiofrequency (Bprf) as a Mechanism of Bio-Stimulation for the Treatment of Vulvo-Vaginal Laxity: A Novel Approach. International Journal of Clinical and Experimental Medicine Research, 5(3), 367-376.

DOI: 10.26855/ijcemr.2021.07.022
\end{abstract}

Received: June 5, 2021

Accepted: June 30, 2021

Published: July 15, 2021

*Corresponding author: Ricardo Galvan, Md Dermatologist, Laser Specialist, Guadalajara, Mexico.

Email: doctorricardogalvan@hotmail.com

\begin{abstract}
Objective: Vaginal laxity is a symptom of pelvic floor dysfunction, often is a poorly defined condition, demand for treatment is increasing every day, there are available alternatives with energy-based devices (EBD) such as laser and radiofrequency. Our aim is to demonstrate the impact of Bipolar Radiofrequency (BPRF) on the pelvic floor for aesthetic and functional indications. Methods: A 12 week prospective evaluation was performed to 15 patients of a primary urogynecology unit that met inclusion criteria. Validated questionaires were used before and after BPRF protocol. Results: important changes in the scores of vulvo vaginal laxity questionnaires and perineometry values after BPRF were found No adverse events from the protocol were reported. Conclusions: BPRF treatment was well tolerated and showed a 3-month safety profile in this pilot study, changes in the scores of validated questionnaires are related to subjective improvement in vaginal tightness, these findings warrant longer follow up, and open a new frontier that can improve quality of life.
\end{abstract}

\section{Keywords}

Vaginal Laxity, Energy Based Devices, Radiofrequency

\section{Introduction}

Vaginal laxity is one of the most common symptoms of pelvic floor dysfunction, some studies have investigated its presence among urogynecology patients documenting a prevalence of $24 \%-38 \%$ but no information is available regarding its incidence in the general population [1]. There is a general agreement about the association of vaginal laxity with pregnancy and childbirth [2]. The association between vaginal laxity and measures of the elevator ani muscle hyper distensibility (genital hiatus + perineal body and hiatal area on Valsalva) has been demonstrated in previous studies [3].

There is functional evidence of muscle injury after childbirth, related to a decrease in pelvic floor muscle strength that is associated with women's parity, in the immediate postpartum period and even a decade after childbirth [4].

Although vaginal laxity is poorly characterized in the medical literature, new proposed terminology appears in the scientific literature, like genitopelvic laxity, which refers to medical/functional disorders that can have a signif- 
icant impact on a woman's sexual self-esteem and sexual life [2]. Ostrensky et al. consider a sensation of wide vagina as the overall increased size of the vagina causing decreased sexual function. Vaginal laxity is identified as the absence of texture due to vanished vaginal rugae that may be related to loss of quality in sexual function [4]. Data from the literature suggest that vaginal winds due to laxity are frequent and seriously impact quality of life of female patients [5].

Vaginal laxity can be considered a highly subjective complaint, anyhow there are tools available to understand patient's feelings related to the pelvic floor health, such as validated questionnaires and quality of life scales. In contrast, there are available tools to measure objectively the strength of pelvic floor in centimeters of water such as the perineometer [6], and tactile imaging (elastography), a useful tool to measure the strength of pelvic floor which helps to understand the relative contribution of pelvic floor muscles into vaginal laxity and prolapse development [7].

Traditional therapeutic options for vaginal laxity as pelvic floor physiotherapy and biofeedback remain to be the gold standard strategies for this condition, unfortunately a vast majority of patients do not have an important improvement either subjectively or objectively, also some of them do not comply with the treatment protocols, Therefore, new strategies with the use of energy-based devices appear as alternatives, the first report by Pardo et al., despite, that is a surgical procedure described an important improvement of vaginal laxity after performing a modified colpoperineoplasty procedure and enhanced sexual gratification in the studied population [8].

Subsequently, energy-based devices are used for the performance of non-invasive or minimally invasive procedures such as $\mathrm{CO}_{2}$ laser and Erbium Yag to improve vaginal relaxation syndrome [9].

Salvatore et al., investigated the effects of fractional $\mathrm{CO}_{2}$ laser on sexual function and satisfaction on a 12-week follow-up study, founding significant improvement in vaginal laxity scores [9].

Subsequently, an experimental observation of the upregulation control of matrix metalloproteinase-2 (MMP-2), well known as an interstitial collagenase, and its relation to the balance between production and degradation of collagen inside endopelvic fascia after a thermal stimuli with fractional $\mathrm{CO}_{2}$ laser, was evaluated [10]. Regenerative mechanisms identified as early as 1 hour after one fractional $\mathrm{CO}_{2}$ laser application with circular polarizing microscopy applied to Picrosirius red stained sections from vaginal mucosa biopsies support the renewal of the extracellular matrix and the formation of new vessels and papillae, developing the stimulation and thickening of the epithelium stimulating the thickening of the epithelium [11].

Non-ablative radiofrequency (RF) has been used as a minimally invasive surgical technique for vaginal laxity, devices emit focused electromagnetic waves against tissue impedance, generating heat inside the tissue in the range of $40-45^{\circ} \mathrm{C}$ to stimulate activation of fibroblasts and synthesis of new collagen and elastin fibers by heat shock proteins mediated inflammatory cascade [12].

There are currently quite a fair number of devices in the market that offer RF-based intravaginal and vulvar treatments, they do not require anesthesia, treatment protocols are usually less than 25 minutes. Patients report a tolerable sensation of warmth without downtime [13].

We consider important to differentiate, the technological aspects of this devices, specifically regarding the polarity.

Monopolar RF has a contact electrode with the tissue and another electrode that usually is very far away from it, usually a plaque which is in contact with hips or feet. This way is usually considered an electro-coagulator because it is permitting a higher flux of current, necessary for vaporizing the tissue. In this methodology is very difficult to foresee where the current is passing through and potentially more dangerous, in contrast Bipolar RF is emitted between two electrodes that usually are very close (maximum distance few millimiters), the electrical current is passing from one electrode to the other and there are no possibilities to go around the body, in this way we're absolutely sure that current is confined just between the electrodes [12].

Millheiser et al. conducted a pilot study in 24 women (aged 25-44 years) using reverse gradient RF energy $\left(75-90 \mathrm{~J} / \mathrm{cm}^{2}\right)$, delivered through the vaginal mucosa. Post-treatment assessments were at 10 days and 1, 3, and 6 months. Results from the study showed that after 1-month, post-treatment self-reported vaginal tightness significantly improved in $67 \%$ of subjects and in $87 \%$ at 6 months. Mean sexual function scores also improved, and no adverse events were reported [14].

Leibaschoff et al., found important changes in Vaginal laxity and sexual satisfaction after monopolar radiofrequency treatment, with a follow up of 8 weeks in 20 voluntary subjects [15].

Alinsod et al., found in a prospective study of 25 subjects that 23 of them had an improvement of $50 \%$ of reduction in time to orgasm and also noted a significant vaginal tightening effects, increased vaginal moisture, and improved vulvar and clitoral sensitivity. These patients were treated using the ThermiVa device for 5 min per zone 
[16].

Regarding BPRF, there is limited data regarding its safety and effectiveness. Dayan et al., found an important improvement in maximal pelvic floor contraction in 31 patients after three sessions of BPRF protocol.

\section{Methods}

An experimental prospective descriptive evaluation was performed between August and November 2019 to patients of a primary urogynecology unit in obstetrics and gynecology department at Hospital Universitario San Jorge Pereira Colombia. A total of 15 patients with an average age of 32 years, multiparous and without overweight as shown in the demographic distribution (Table 1) were seen for a standardized BPRF protocol, inclusion criteria were patients complaining about vaginal winds, water entrapment, loss of friction during sexual intercourse and labia majora laxity. Patients with previous pelvic organ prolapse surgery, Pelvic organ prolapse POP Q> 1, smokers, non-compliant with protocol and other co morbidities were excluded from the study group (Table 2).

Table 1. Demographics

\begin{tabular}{|c|c|c|c|}
\hline \multicolumn{3}{|c|}{ Items } & $\mathrm{n}=15$ \\
\hline \multicolumn{3}{|c|}{ Age } & $32 \pm 4.2$ \\
\hline \multicolumn{3}{|c|}{ Parity } & 2.0 \\
\hline \multicolumn{3}{|c|}{ Past History of cesarean section } & 5 (33.3) \\
\hline \multicolumn{3}{|c|}{ Vaginal delivery } & $10(66.6)$ \\
\hline \multicolumn{3}{|c|}{ BMI } & $23 \pm 1.98$ \\
\hline \multirow{3}{*}{ Pelvic Floor Examination } & \multirow{3}{*}{ Pelvic Organ Prolapse POP Q } & No Distopy & $12(80)$ \\
\hline & & Stage 0 & $3(20)$ \\
\hline & & Genital Hiatus Distance & $3.2 \pm 1.4$ \\
\hline
\end{tabular}

Table 2. Inclusion / Exclusion criteria

\begin{tabular}{|c|c|}
\hline INCLUSION CRITERIA & EXCLUSION CRITERIA \\
\hline $\begin{array}{ll}\text { - } & \text { Volunteers complaining about vaginal winds } \\
\text { - } & \text { Volunteers complaining about water entrapment } \\
\text { - } & \text { Volunteers complaining about loss of friction } \\
\text { - } & \text { Volung sexual intercourse } \\
& \text { Volunteers complaining about labia majora laxity }\end{array}$ & $\begin{array}{l}\text { - Volunteers withPOP Q> } 1 \\
\text { - Volunteers with a history of a recto-vaginal or vesico-vaginal } \\
\text { fistula } \\
\text { - Volunteers unable to follow-up for a period of } 9 \text { months' } \\
\text { post-treatment } \\
\text { cytology } \\
\text { cynormal HPV DNA test and subsequent abnormal liquid based } \\
\text { - Volunteers with Undiagnosed uterine bleeding } \\
\text { treatment for the management of their vaginal laxity }\end{array}$ \\
\hline
\end{tabular}

Validated scales such as Sexual Satisfaction Questionnaire SSQ, Vaginal Laxity Questionnaires VLQ, ICQ-SF, Vagina Flatus Score VFS, Modified Oxford Grading MOG and visual analogue scale VAS for global satisfaction and perineometry values were measured before and after protocol with BPRF (Tables 3, 4, 5, 6, 7).

Table 3. Sexual Satisfaction Questionnaire SSQ

\begin{tabular}{|l|}
\hline \multicolumn{2}{|c|}{ SEXUAL SATISFACTION QUESTIONNAIRE (SSQ) } \\
\hline Please rate your sexual satisfaction from vaginal intercourse since childbirth using the 6-level ordered scale: \\
$\square$ None \\
$\square$ Poor \\
$\square$ Fair \\
$\square$ Good \\
$\square$ Very Good \\
Excellent
\end{tabular}


Table 4. Vaginal Laxity Questionnaires VLQ

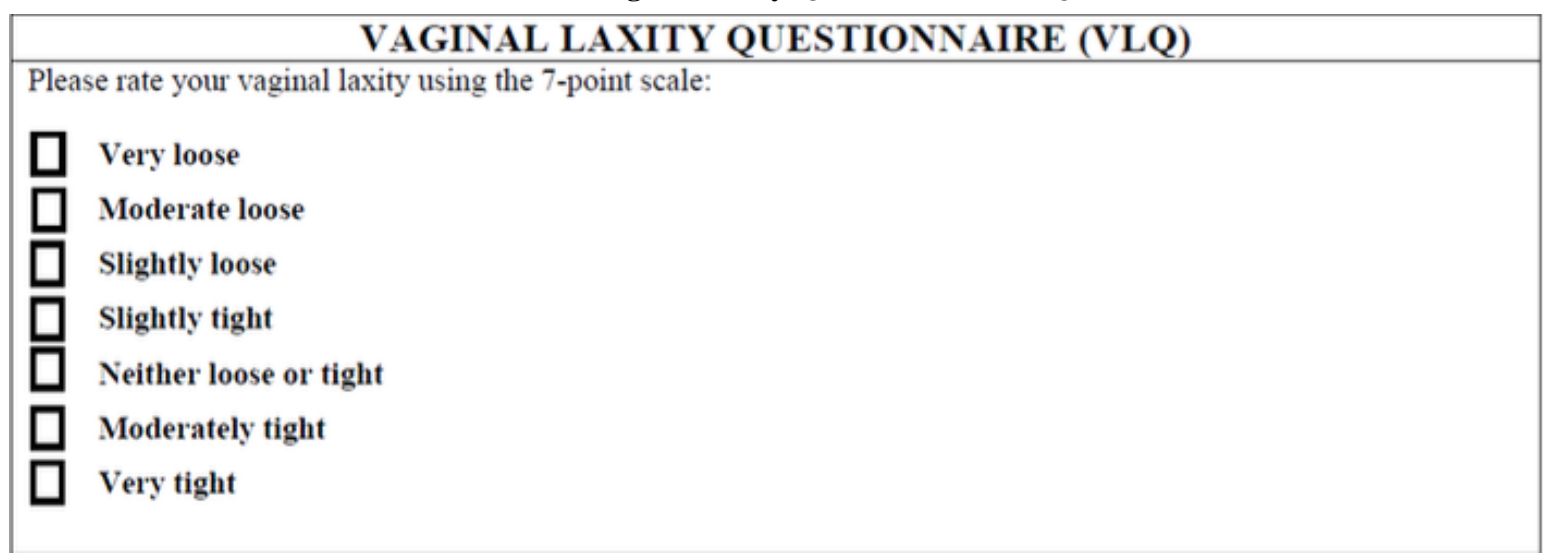

Table 5. ICQ-SF

Many people leak urine some of the time. We are trying to find out how many people leak urine, and how much this bothers them. We would be grateful if you could answer the following questions, thinking about how you have been, on average, over the PAST FOUR WEEKS.

1 Please write in your date of birth:

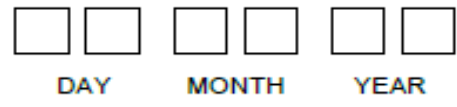

2 Are you (tick one):

Female $\square$ Male

3 How often do you leak urine? (Tick one box)

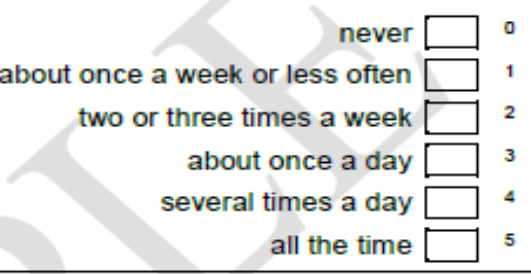

4 We would like to know how much urine you think leaks.

How much urine do you usually leak (whether you wear protection or not)? (Tick one box)

$\begin{aligned} \begin{array}{r}\text { none } \\ \square\end{array} & 0 \\ \text { a small amount } \square & 2 \\ \text { oderate amount } \square & 4 \\ \text { a large amount } \square & 6\end{aligned}$

5 Overall, how much does leaking urine interfere with your everyday life? Please ring a number between 0 (not at all) and 10 (a great deal)

\begin{tabular}{|ccccccccccc}
0 & 1 & 2 & 3 & 4 & 5 & 6 & 7 & 8 & 9 & $\begin{array}{l}10 \\
\text { a great deal }\end{array}$ \\
not at all & & & & & & & & & &
\end{tabular}

ICIQ score: sum scores 3+4+5

6 When does urine leak? (Please tick all that apply to you)

$$
\begin{array}{r}
\text { never - urine does not leak } \\
\text { leaks before you can get to the toilet } \\
\text { leaks when you cough or sneeze } \\
\text { leaks when you are asleep }
\end{array}
$$

leaks when you are physically active/exercising leaks when you have finished urinating and are dressed

leaks for no obvious reason

leaks all the time

Thank you very much for answering these questions.

Copyright $\odot$ "ICIQ Group" 
Table 6. Vaginal Flatus Score VFS

Vaginal Flatus Score

\section{How often to you feel wind or an air bubble coming out of your vagina (also known as vaginal flatus)?}

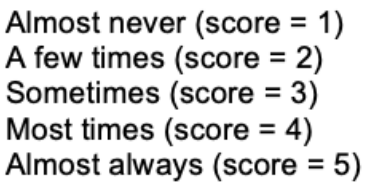

Table 7. Modified Oxford Grading MOG

Modified Oxford Grading

- Score is obtained by digital palpation of pelvic floor muscles

- One finger is placed on the posterior vaginal wall approximately 2 $\mathrm{cm}$ deep into the vaginal. The patient is asked to try and 'squeeze' the finger' in order to assess the strength of their pelvic floor musculature

\begin{tabular}{|l|l|}
\hline 0 & No contraction \\
\hline 1 & Flicker \\
\hline 2 & Weak \\
\hline 3 & Moderate (with lift) \\
\hline 4 & Good (with lift) \\
\hline 5 & Strong (with lift) \\
\hline
\end{tabular}

Patients recruited for this study were volunteers from the Urogynaecology unit. They offered to participate prior to obtaining informed consent and a written authorization to be included in their medical records according to Helsinki declaration, Belmont report, CIOMS rules, GPC/ICH and 008430 resolution of Colombia government stabilized in 4th October 1993. Present investigation was considering as minimal risk or beyond minimal risk as follows:

- Adjust and briefly explain the ethical principles that warrant investigation according to international standards.

- Based on previously conducted experiments on animals in laboratories and other scientific facts that show a secure intervention in humans.

- Clearly express the risks and security guarantees to participants.

- Having the written informed consent of research subjects or their legal representative.

- Relate the experience of researchers and the responsibility of a health entity.

\section{Protocol}

After informed consent signature, patients are placed in dorsal lithotomy on the exam table, fair amount of clear water-based warm gel was used as a conductive medium. Vaginal treatment was performed dividing the vagina in 4 quadrants and three thirds distal, middle and proximal (Figure 1) and vulvar treatment was applied in three different anatomical subunits: labia majora, crural fold and interlabial crease (Figure 2).
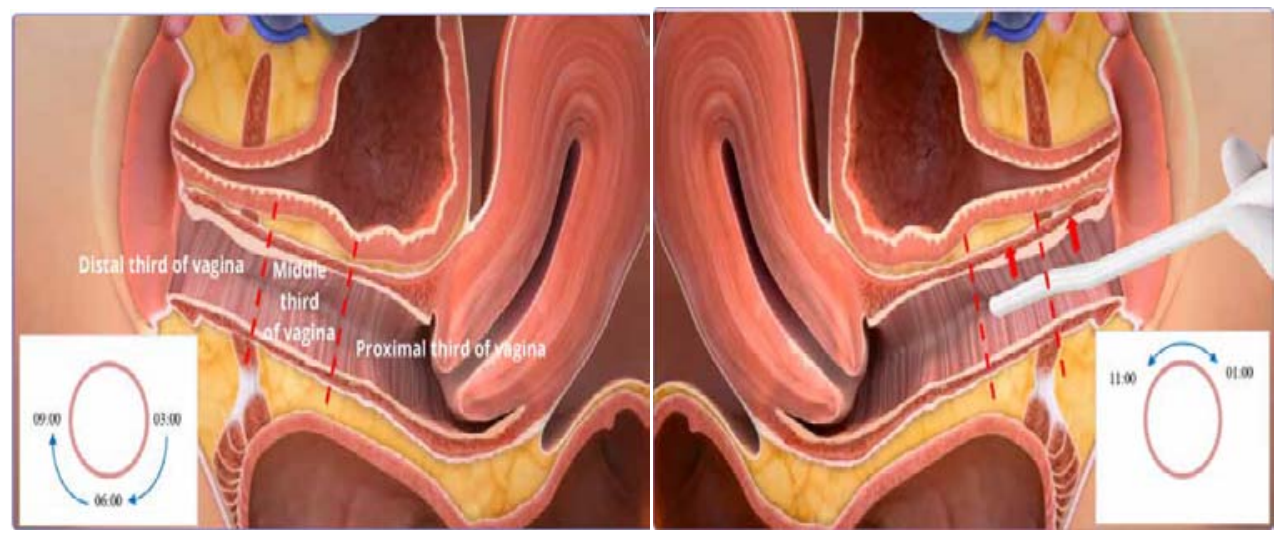

Figure 1. Vaginal application BPRF. 


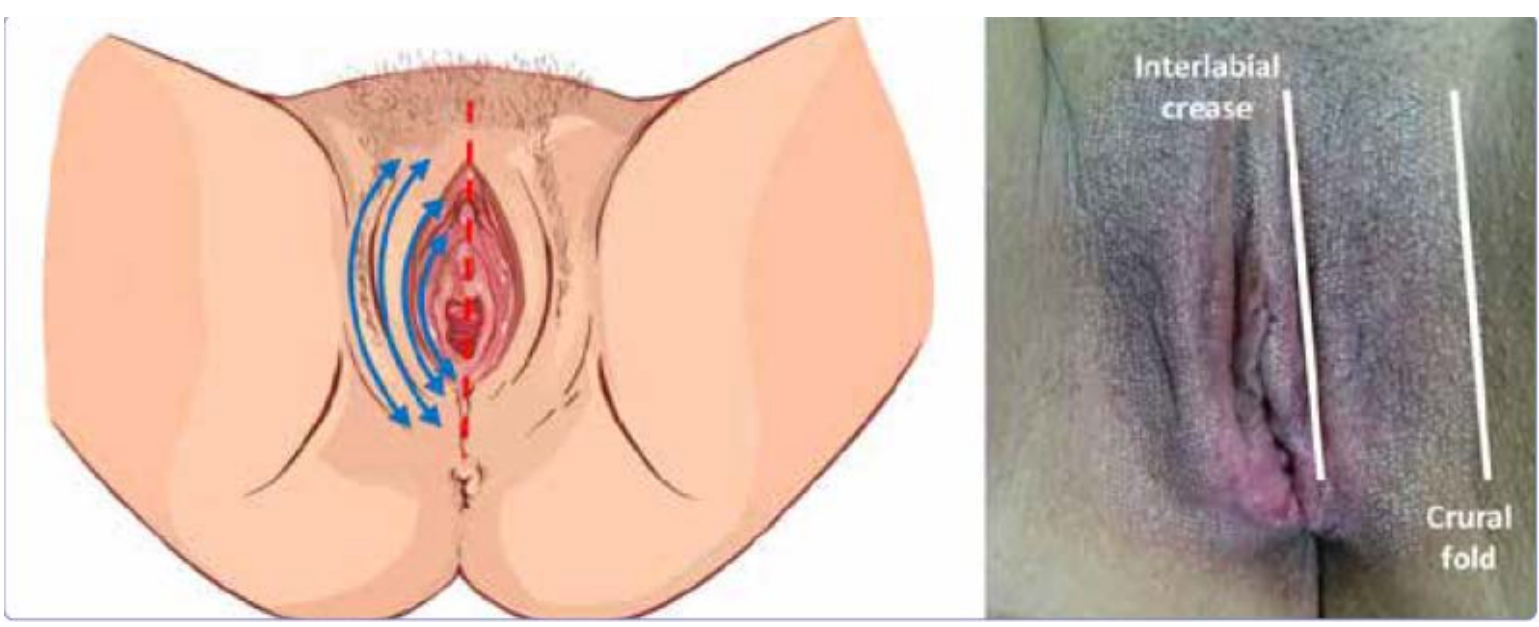

Figure 2. Vulvar application BPRF.

BPRF energy was applied to the labia majora using a special probe it was passed back and forth slowly with wide movements over each treated area for 16 minutes to Vulva and 24 minutes to Vagina. Power from the RF source was set in a range of 35 to 40 watt, the temperature setting was $42^{\circ} \mathrm{C}$ to $45^{\circ} \mathrm{C}$ depending on patient tolerance, schedule visits were set at 2-3 week interval for vulvar and vaginal treatments (Table 8). For touch up, sessions should be scheduled not before 6 months or after 12 months based on first protocol, with time touch up treatment of 8 min vagina 8 min vulva total 16 min session (Table 8).

Perineometry values were measured with the manometric biofeedback perineometer (Kinemax Products Chile). The perineometer is a vaginal probe that measures pelvic floor muscle strength in centimeters of water. It has been shown to be valid, with accurate readings.

Table 8. Parameters per zone

\begin{tabular}{|c|c|c|c|c|c|c|}
\hline Area & Indication & Power & Temperature & Time & $\begin{array}{c}\text { Intervals / } \\
\text { sessions }\end{array}$ & Observations \\
\hline Vulva & Laxity & 40 watt & $41-42$ & 8 min per & $\begin{array}{lr}\text {-Sessions } & \text { every } \\
2 \text { weeks } & \\
-4-6 & \text { sessions } \\
\text { needed r } & \text { to } \\
\text { improve vulvar } \\
\text { laxity }\end{array}$ & $\begin{array}{l}\text { Long sweeping } \\
\text { motions keep } \\
\text { temperature over } \\
40 \text { degress } \\
\text { from the crural } \\
\text { fold to the } \\
\text { interglacial } \\
\text { crease }\end{array}$ \\
\hline Vulva & Atrophy & 35 watt & $40-42$ & 6 min per & $\begin{array}{l}\text {-Sessions every } \\
3-4 \text { weeks } \\
-4-6 \text { sessions } \\
\text { needed to } \\
\text { improove vulvar } \\
\text { atrophy }\end{array}$ & $\begin{array}{l}\text { Long sweeping } \\
\text { motions keep } \\
\text { temperature over } \\
40 \text { degress } \\
\text { from the crural } \\
\text { fold to the } \\
\text { interglacial } \\
\text { crease }\end{array}$ \\
\hline Vagina & Laxity & $35-40$ & Four. Five & $\begin{array}{l}6 \text { min per } \\
\text { quadrant }\end{array}$ & $\begin{array}{l}\text {-Sessions every } \\
3 \text { weeks } \\
-4-6 \text { sessions } \\
\text { needed to } \\
\text { improove } \\
\text { vaginal laxity }\end{array}$ & $\begin{array}{ll}\text { From apex of } \\
\text { vagina } & \begin{array}{l}\text { to } \\
\text { himeneal ring }\end{array}\end{array}$ \\
\hline Vagina & Atrophy & 35 & Four. Five & $\begin{array}{l}6 \text { min per } \\
\text { quadrant }\end{array}$ & $\begin{array}{l}\text {-Sessions every } \\
4 \text { weeks } \\
-4-6 \text { sessions } \\
\text { needed to } \\
\text { improove } \\
\text { vaginal laxity }\end{array}$ & $\begin{array}{ll}\text { From apex of } \\
\text { vagina } & \text { to } \\
\text { himeneal ring }\end{array}$ \\
\hline Vagina & Mild sui / oab & 40 & Four. Five & 10 minutes & & $\begin{array}{l}\text { Mid urethra and } \\
\text { anterior } \\
\text { compartments } \\
\text { only }\end{array}$ \\
\hline $\begin{array}{l}\text { Clitoral } \\
\text { hood }\end{array}$ & $\begin{array}{l}\text { Orgasmic } \\
\text { dysfunction }\end{array}$ & 40 & 42 & $6 \underset{\text { side }}{\min \text { per }}$ & & $\begin{array}{lr}\text { From fosita } \\
\text { navicularis to } \\
\text { clitoral frenulum }\end{array}$ \\
\hline Vestibule & $\begin{array}{l}\text { Chronic vulvar } \\
\text { fissure }\end{array}$ & 40 & $42-45$ & $7 \mathrm{~min}$ & & $\begin{array}{lr}\text { From distal } \\
\text { vagina } \\
\text { perineal body }\end{array}$ \\
\hline
\end{tabular}

Validated scales such as Sexual Satisfaction Questionnaire SSQ, Vaginal Laxity Questionnaires VLQ, ICQ-SF, Vaginal Flatus Score VFS, Modified Oxford Grading MOG and visual analogue scale VAS for global satisfaction were filled by the patients before and after (BPRF) protocol. 


\section{Results}

The present study included a small population of 15 patients as it was a pilot and experimental study that is useful as a starting point for future studies.

Overall satisfaction at last session performed (day 100) showed that $90 \%$ of patients (13.5 of 15) were "satisfied" with the improvement of vaginal tone after the treatment protocol (Table 9).

Table 9. Scores before and after protocol

\begin{tabular}{ccc}
\hline Score & Baseline & End of Protocol \\
\hline Sexual Satisfaction Questionnaire SSQ & 2.53 & 4.93 \\
Vaginal Laxity Questionnaire VVLQ & 2.46 & 4.66 \\
ICQ-SF & 14.5 & 9.37 \\
\hline
\end{tabular}

As general results of the application of the SEXUAL SATISFACTION QUESTIONNAIRE, it was evidenced for the unit of analysis in $100 \%$ patients $(n=15)$ that met the criteria, and a baseline analysis was performed by the following categories POOR 47\% $(n=7)$ and FAIR $(n=8)$; and after the end of the protocol, the following results were obtained GOOD 34\% ( $n=5)$, VERY GOOD 40\% ( $n=6)$, EXCELLENT 27\% (4), observing an improvement in $100 \%(n=15)$ of the patients regarding sexual function.

Regarding to VAGINAL LAXITY QUESTIONARY; in their baseline state before the protocol the patients scored VERY LOSE 7\% $(n=1)$, MODERATE LOSE 40\% $(n=6)$, SLIGHT LOOSE 53\% $(n=8)$. After a 100-day follow-up, it was verified that at the end of the protocol there were significant changes in the vulvovaginal tissue tone and improvement of its contusion passing to SLIGHTLY LOOSE 6.7\% $(n=1)$, SLIGHTLY TIGHT 34\% $(n=$ 5), NEITHER LOOSE OR TIGHT 47\% ( $n=7)$, MODERATELY TIGHT 2\% $(n=1)$ (Figures 3 and 4).
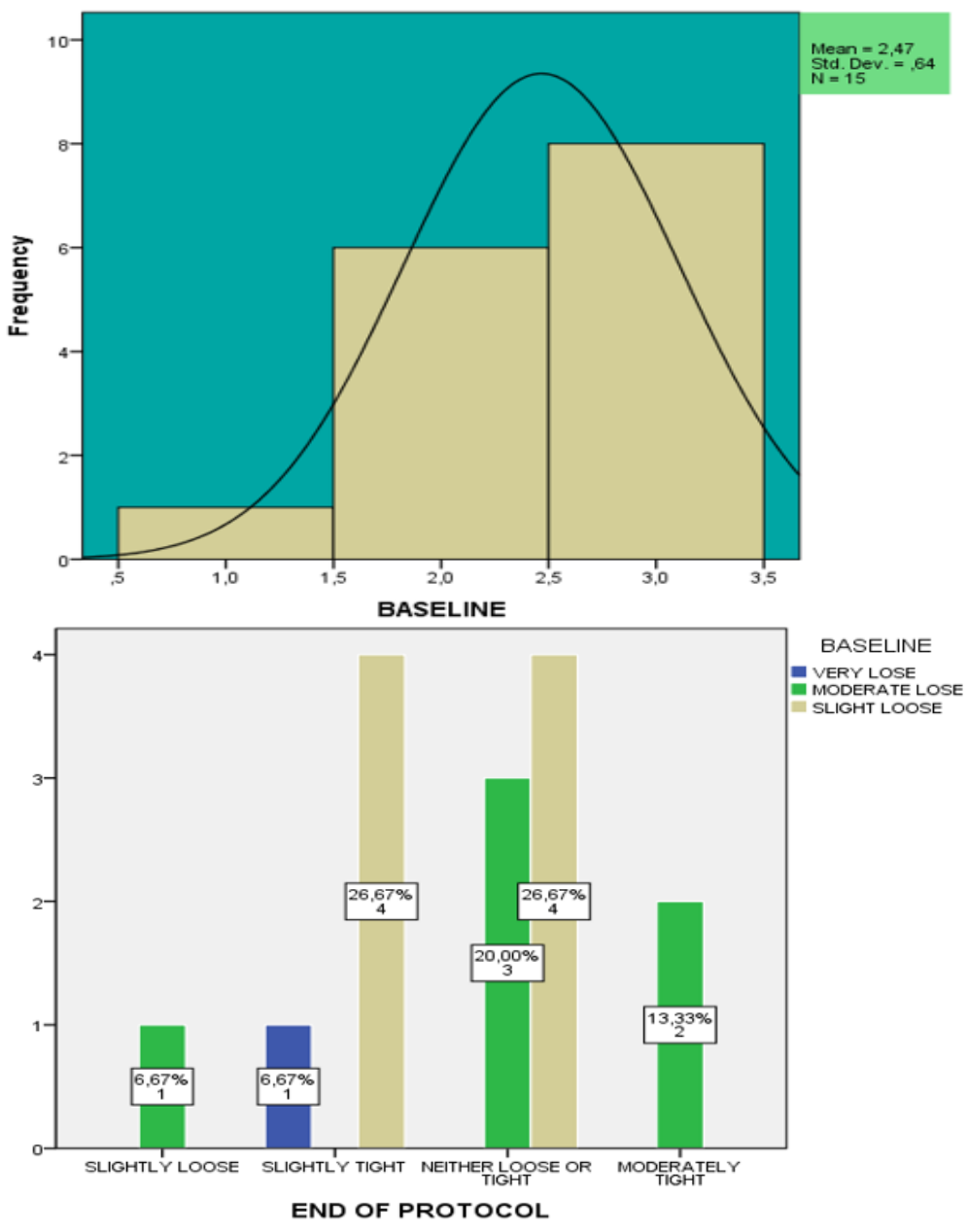

Figures 3 \& 4. Vaginal Laxity Questionnaire. 
60\% (8 of 15) patients who met criteria for SUI, reported to have $70 \%$ improvement in SUI, and it was correlated with important changes in the scores of ICQ-SFin which there was an improvement in $50 \%$ regarding interference with quality of life at a baseline state of an average 14.5.

When comparing the measurement of the VAGINAL FLATUS SCORE, in baseline it was obtained as follows: A FEW TIMES 7\% $(n=1)$, SOMETIMES 40\% $(n=6)$, MOST TIMES 40\% $(n=6)$, ALMOST ALWAYS $14 \%(n=2)$ and after follow-up and completion of the control, the majority of patients in the A FEW TIMES category $54 \%(\mathrm{n}=$ 8) were distributed, ALMOST NEVER 34\% $(n=5)$, SOMETIMES $14 \%(n=2)$ (Figure 5).

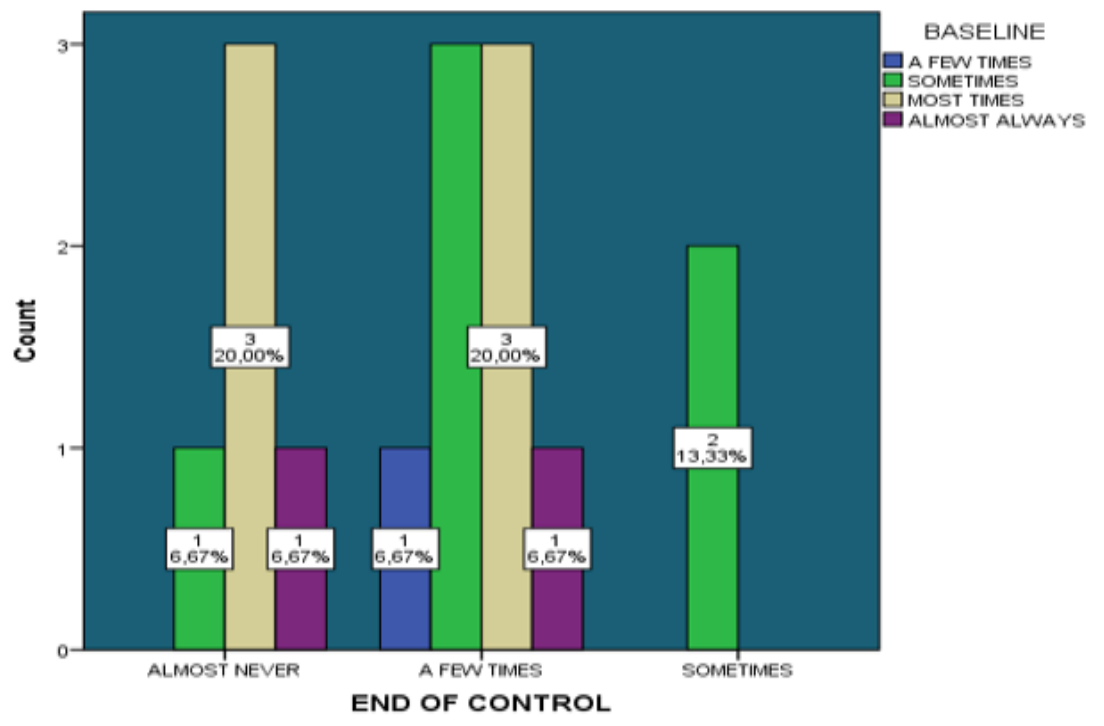

Figure 5. Vaginal Flatus Score.

In the exploratory analysis with the MODIFIED OXFORD GRADING scale, applied to $100 \%$ of the study population ( $\mathrm{n}=15)$, an initial state GOOD (WITH LIFT) 10\% ( $=1$ ), MODERATE (WITH LIFT) 45 was found\% 8 $(n=7)$, WEAK $45 \%(n=7)$, also at the end of the control after the follow-up of the patients, a classification was again made according to their symptom resulting in GOOD (WITH LIFT) 50\% ( $\mathrm{n}=7$ ), MODERATE (with lift) 30\% $(n=5)$, STRONG (with lift) $20 \%(n=3)$, showing improvement in $80 \%$ to $90 \%$ of patients after the intervention.

Visual analogue scale VAS for global satisfaction went from 7.2 to 2.6 regards to general involvement due to the symptoms related to vulvovaginal laxity.

None of the 15 patients included in the protocol presented adverse events or unwanted symptoms related to the treatment.

\section{Discussion}

This research allowed to know the results of radiofrequency as a treatment for vulvovaginal laxity in a group of women. In this sense, the results allow to outline an encouraging mood regarding the improvement of sexual satisfaction, decrease in vulvovaginal laxity, decrease in vaginal flatus, increase in pelvic floor strength, and decrease in pain sensation according to the analog scale visual.

Radio frequency has been shown to be a technique that provides clinical improvement regarding the effects of vaginal laxity [12]. Studies similar to ours have revealed benefits not only in improving laxity [14] [17] [21], but also, in vaginal lubrication, resolution of anorgasmia [22], and recovery of urinary continence [17] [18] [23].

Regarding sexual satisfaction, an increase of 2.40 points was found, starting from a base 2.53; and scores between "Good" and "excellent" were obtained in 91\% in total, being similar to that reported by various studies in which a significant statistical difference $<0.01$ was found [14] [17], and equal to 0.02 [22], 92.4\% satisfaction rate [23], and 93\% [18] and sexual improvement [19] in this variable among other studies. Although anxiety related to sexual activity was not addressed in our study, it was found that radiofrequency allowed a significant decrease of $9.8 \pm 8$ points during 12 months [21].

Vaginal laxity increased in the patients included in the study by 2.20; the base score being 2.46, by identifying items as "slightly loose", "slightly tight", "neither loose nor tight" and "moderately tight" with a total percentage of 89.7\%; which is similar to what Caruth (2018) referred to in his research [17], as well as by Lalji and Lozanova (2017) who reported 89\% from "very loose” to "moderately tight” [18], by Sekiguchi et al. with a statistically sig- 
nificant difference $(<0.001)$ during 12 months, and finally by Millheiser et al. (2010) with a total improvement of $87 \%$ [14]. This type of results is to be expected since radiofrequency favors collagen contraction, neocolagenesis, angiogenesis and infiltration of the growth factor that allows restoring the elasticity of the vaginal mucosa [24].

The women included reported an improvement in urinary incontinence by $70 \%$, being lower than that reported by Lalji and Lozanova (2017), who in their study document 90\% (18), and Caruth (2018) reported significant improvement [17].

In the vaginal flatus score, it is striking that before treatment items such as "most of the time" and "almost always" correspond to $54 \%$, which changed at the end of the treatment to "sometimes in the same percentage". denoting this a great improvement. However, this scale was not found in the referred studies, which is why its comparison was not possible.

The previous behavior also occurs with the Modified Oxford Scale, which initially presented $10 \%$ in the item "Good", increasing by $40 \%$ at the end of the treatment, also finding a criterion that was not initially present, which was "Strong” with $20 \%$.

In the same way, the visual analog pain scale behaved, presenting a noticeable improvement by decreasing 4.6 points, the base score being 7.2, the same phenomenon happening with the referred studies, which did not use this scale. However, a study that addressed atrophic vaginitis and dyspareunia, shows an improvement in pain as an effect of radiofrequency [22].

The women participating in this research did not present adverse events, and it is common that related studies do not mention the presence of these. However, it is documented that vaginal discharge, pain, discomfort, erythema and edema have been observed with rapid resolution [24] [25].

The limitations found in the development of this research are limited to the small proportion of studies that address radio frequency as a treatment for vulvovaginal laxity, which despite allowing to affirm that it generates great benefits in genital functionality in women, requires various studies that further strengthen the therapeutic profile of this technique through scientific evidence. Another limitation that can be elucidated is the temporality of the tension and functional effect of radiofrequency, being aspects that must be studied in more depth to give this technique a higher status than the one it currently has given its proven effectiveness by being able to fully know its therapeutic profile and maintenance of the physiological effect in women.

\section{Conclusion}

Vaginal laxity is a condition that requires attention from pelvic floor health providers, it could affect quality of life and sexual life since it can be related to loss of friction during sexual intercourse, vaginal gases and impairment of quality of life.

An important percentage of patients could have major changes with noninvasive techniques such as BPRF, with improvement of global sexual satisfaction, subjective improvement of vaginal laxity and improvement of vaginal flatus.

This experimental protocol demonstrates the safety and efficacy of using this novel energy device for Vulvo/Vaginal laxity and stress urinary incontinence in a group of voluntary patients.

We consider this novel alternative as safe, easy to apply with minimal risk, the bipolarity characteristics make it safer and with a controlled depth of heat penetration to the endopelvic fascia.

This study showed that strengthening the pelvic floor, reducing pain during intercourse, eliminating vaginal flatus, urinary continence and improving the perception of sexual satisfaction through this technique, notably increases the quality of personal and family life of women with vulvovaginal laxity, by allowing patients to improve or regain comfort, security, confidence and performance in their sexual life.

\section{References}

[1] Pauls RN, Fellner AN DG. (2012). Vaginal laxity: a poorly understood quality of life problem; a survey of physician members of the International Urogynecological Association (IUGA). Int Urogyn J., 2012, 23: 1435-48.

[2] Newman R CP. (2018). Genito Pelvic Vaginal Laxity: Classification, Etiology, Symptomatology, and Treatment Considerations. Curr Sex Heal Rep., 2018, 10: 222-36.

[3] Dietz HP, Stankiewicz M, Atan IK, Ferreira CW SM. (2018). Vaginal laxity:what does this symptom mean? Int Urogyn J., 2018, 29(5): 723-8.

[4] Adam Ostrzenski. (2011). An acquired sensation of wide/smooth vagina: a new classification. Eur J Obs Gynecol Reprod Biol., 2011, 158: 97-100. 
[5] Amarenco G, Turmel N, Chesnel C, Mezzadri M, Le Breton F, Charlanes A HC. (2019). Vaginal gas: Review. Prog Urol., 2019, 29(17): 1035-40.

[6] Frawley H, Galea M, Phillips B, Sherburn M BK. (2006). Reliability of pelvic floor muscle strength assessment using different test positions and tools. Neurourol Urodyn, 2006, 25: 236-42.

[7] Heather van Raalte, Vincent Lucente, Vladimir Egorov. (2015). High definition pressure mapping of the pelvic floor muscles during valsalva manever, voluntary muscle contraction and involuntary relaxation. Fem pelv med reconst surg., 2015, 21(5): 149-50.

[8] Pardo JS, Sola VD, Riccia PA, Guiloff EF FO. (2006). Colpoperineoplasty in women with a sensation of a wide vagina. Act Obs Gyn Scan., 2006, 85(9): 1125-7.

[9] Salvatore S, Nappi RE, Parma M, et al. (2015). Sexual function after fractional microablative CO (2) laser in women with vulvovaginal atrophy. Climateric, 2015, 18(2): 219-25.

[10] Filippini M, Del Duca E, Negosanti F, et al. (2017). Fractional CO2 laser: from skin rejuvenation to vulvovaginal reshaping. Photomed Laser Surg., 2017, 35(3): 171-5.

[11] Aimes RT QJ. (1995). Matrix metalloproteinase-2 is an interstitial collagenase. Inhibitor free enzyme catalyzes the cleavage of collagen fibrils and soluble native type I collagen generating the specific 3 / 4- and 1/4-length fragments. J Biol Chem., 1995, 270: 5872-6.

[12] Qureshi AA, Tenenbaum MM MT. Nonsurgical vulvovaginal rejuvenation with radiofrequency and laser devices: a literature review and comprehensive update for aesthetic surgeons. Aesthet Surg J., 18AD, 38(3): 302-11.

[13] Karcher C SN. (2016). Vaginal rejuvenation using energy-based devices. Int J Wom Dermat., 2016, 2: 85-8.

[14] Millheiser LS, Pauls RN, Herbst SJ CB. (2010). Radiofrequency treatment of vaginal laxity after vaginal delivery: nonsurgical vaginal tightening. J Sex Med., 2010, 7(9): 3088-95.

[15] Leibaschoff G, Izasa PG, Cardona JL, Miklos JR MR. (2016). Transcutaneous temperature controlled radiofrequency (TTCRF) for the treatment of menopausal vaginal/genitourinary symptoms. Surg Technol Int., 2016, 29: 149-59.

[16] Red M. Alinsod. (2016). Temperature controlled radiofrequency for vulvovaginal laxity. Lasers Surg Med., 2016, 48(7): 641-5.

[17] Jeffrey C. Caruth. (2018). Evaluation of the safety and efficacy of a novel radiofrequency device for vaginal treatment. Surg Technol Int., 2018, 32: 145-9.

[18] Lalji S LP. (2017). Evaluation of the safety and efficacy of a monopolar nonablative radiofrequency device for the improvement of vulvovaginal laxity and urinary incontinence. J Cosmet Dermat., 2017, 16: 230-4.

[19] Red M. Alinsod. Transcutaneous temperature controlled radiofrequency for orgasmic dysfunction. Lasers Surg Med.2., 2016, 48: 641-5.

[20] Vicariotto F, DE Seta F, Faoro V RM. (2017). Dynamic quadripolar radiofrequency treatment of vaginal laxity/menopausal vulvo-vaginal atrophy: 12-month efficacy and safety. Minerva Ginecol., 2017, 69: 342-9.

[21] Sekiguchi Y, Utsugisawa Y AY, et al. (2013). Laxity of the vaginal introitus after childbirth: nonsurgical outpatient procedure for vaginal tissue restoration and improved sexual satisfaction using low-energy radiofrequency thermal therapy. J Womens Heal., 2013, 22: 775-81.

[22] Alinsod RM. (2015). Transcutaneous temperature controlled radiofrequency for atrophic vaginitis and dyspareunia. J Minim Invasive Gynecol., 2015, 22: 226-7.

[23] Kamilos MF BC. (2017). New therapeutic option in genitourinary syndrome of menopause: pilot study using microablative fractional radiofrequency. Einstein, 2017, 15: 445-51.

[24] Preminger BA, Kurtzman JS DE. (2020). A Systematic Review of Nonsurgical Vulvovaginal Restoration Devices: An Evidence-Based Examination of Safety and Efficacy. Plast Reconstr Surg., 2020, 146 (5): 552-64.

[25] Fistonić I, Turina ISB, Fistonić N MI. (2016). Short time efficacy and safety of focused monopolar radiofrequency device for labial laxity improvement-noninvasive labia tissue tightening: A prospective cohort study. Lasers Surg Med., 2016, 48: 254-9. 